\title{
Tizanidine exerts anti-nociceptive effects in spared nerve injury model of neuropathic pain through inhibition of TLR4/NF-kB pathway
}

\author{
WANMIN PEI ${ }^{1}$, YI ZOU ${ }^{2}$, WENTING WANG ${ }^{2}$, LAI WEI $^{2}$, YUAN ZHAO $^{2}$ and LI LI $^{1}$ \\ ${ }^{1}$ Department of Anesthesia, Second Xiangya Hospital, Central South University, Changsha, Hunan 410011; \\ ${ }^{2}$ Department of Anesthesia, People's Hospital of Hunan Province, Changsha, Hunan 410005, P.R. China
}

Received December 19, 2016; Accepted February 12, 2018

DOI: $10.3892 / \mathrm{ijmm} .2018 .3878$

\begin{abstract}
Recently, $\alpha 2$-adrenoceptors ( $\alpha 2$-AR) agonists have been shown to have anti-nociceptive effects and thus may become a promising therapeutic strategy for neuropathic pain. tizanidine is a highly selective $\alpha 2$-AR agonist, but the effect mechanism of tizanidine in neuropathic pain remains largely unknown. The present study investigated whether tizanidine has anti-nociceptive effects in spared nerve injury (SNI) model of neuropathic pain in rats, as well as explored the underlying molecular mechanism. We found that the rats in SNI group showed significantly higher mechanical and thermal hyperalgesia, accompanied with increased production of proinflammatory cytokines including interleukin-1 $\beta$ (IL-1 $\beta$ ), IL-6 and tumor necrosis factor- $\alpha$ (TNF- $\alpha$ ), as well as the activation of Toll-like receptor 4 (TLR4)/nuclear factor- $\kappa \mathrm{B}$ (NF- $\kappa \mathrm{B})$ signaling. PDTC, an inhibitor of TLR4/NF- $\kappa B$ signaling, could significantly attenuate the SNI-induced mechanical and thermal hyperalgesia and the production of proinflammatory cytokines. Moreover, treatment with tizanidine also attenuated the SNI-induced mechanical and thermal hyperalgesia, suppressed production of the proinflammatory cytokines, and inhibited the activation of TLR4/NF- $\mathrm{BB}$ pathway, which could be reversed by pretreatment with BRL44408, a selective $\alpha 2-A R$ antagonist. Taken these findings together, we demonstrated that tizanidine has anti-nociceptive effects on neuropathic pain via inhibiting the production of proinflammatory cytokines through suppressing the activation of TLR4/NF- $\mathrm{BB}$ p65 signaling pathway.
\end{abstract}

Correspondence to: Professor Li Li, Department of Anesthesia, Second Xiangya Hospital, Central South University, 139 Renmin Middle Road, Changsha, Hunan 410011, P.R. China

E-mail: xiangyalili@aliyun.com

Professor Yuan Zhao, Department of Anesthesia, People's Hospital of Hunan Province, 61 Jiefangxi Road, Changsha, Hunan 410005, P.R. China

E-mail: 316890579@qq.com

Key words: tizanidine, anti-nociceptive, spared nerve injury, inflammatory cytokine, rat

\section{Introduction}

Peripheral neuropathic pain is produced by multiple etiological factors, and spared nerve injury (SNI) is an important model for exploring the cellular and molecular mechanism in peripheral neuropathic pain $(1,2)$. It has been demonstrated that the agonists of $\alpha 2$-adrenoceptors ( $\alpha 2$-AR) have anti-nociceptive effects $(3,4)$. Tizanidine, a derivative of clonidine, is a highly selective agonist of $\alpha 2-\mathrm{AR}$, and has the same effects of clonidine, but the side effects such as low arterial blood pressure and bradycardia are lower $(5,6)$. The primary site of tizanidine effect is the spinal cord (7). Through stimulating the $2 \alpha$ presynaptic receptors, tizanidine could prevent the release of aspartic and glutamic acids (8). As other $\alpha 2$-AR agonists, tizanidine has also been demonstrated to exhibit analgesic effects (9). For instance, tizanidine could reduce postoperative pain and the need for tranquilizers through inhibition of central sensitization (10). However, whether tizanidine exerts anti-nociceptive effects in spared nerve injury model of neuropathic pain has not been previously reported.

Toll-like receptor 4 (TLR4), belongs to TLR family, and is an important transmembrane protein acting as a signal transduction molecule in inflammatory responses $(11,12)$. Stimulation of TLR4 can further activate the nuclear factor- $\mathrm{\kappa B}(\mathrm{NF}-\mathrm{\kappa B})$ signaling $(13,14)$. NF- $\kappa \mathrm{B}$, a key transcriptional factor, is universally expressed in various cells, responsible for regulating the transcription of inflammatory cytokines, including tumor necrosis factor- $\alpha$ (TNF- $\alpha$ ), interleukin-1 $\beta$ (IL-1 $\beta$ ), and IL-6, and thus acts as a key regulator of inflammatory responses $(15,16)$. Under normal conditions, inhibitory NF- $\kappa B$

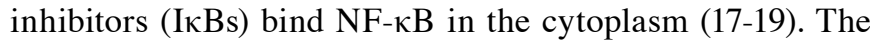
activation of NF- $\kappa B$ begins with the phosphorylation of I $\mathrm{B} \alpha$, which further causes IкB $\alpha$ ubiquitinated and degraded eventually (17-19). Once I $\mathrm{B} \alpha \alpha$ is degraded, NF- $\kappa \mathrm{B}$ translocates to nucleus and promotes the transcription of its target genes, such as IL-1 $\beta$, IL- 6 and TNF- $\alpha$ (17-19). However, the effects of tizanidine on the TLR4/NF- $\mathrm{BB}$-mediated inflammation have not bee previously reported.

This study investigated whether intrathecal administration of tizanidine exhibits anti-nociceptive effects in SNI model of rats, and we also studied the underlying molecular mechanism, involving the activity of TLR4/NF- $\mathrm{BB}$ signaling pathway and the production of inflammatory cytokines. 


\section{Materials and methods}

Animals. This study was approved by Animal Care and Use Committee of People's Hospital of Hunan Province (Changsha, China). Animal experiments were consistent with the National Institutes of Health Guide for the Care and Use of Laboratory Animals. Adult male Sprague Dawley rats (200-250 g) were purchased from Animal Center of Central South University (Changsha, China) and housed in light-controlled $(12 \mathrm{~h}$ dark $/ 12 \mathrm{~h}$ light cycle) and temperature-controlled $\left(22 \pm 2^{\circ} \mathrm{C}\right)$ room with free access to clear water and food.

Surgical procedure of SNI model. Under enflurane (3.0 v\%) anesthesia, an incision was made in the skin on the lateral surface of the thigh of rats. The biceps femoris muscle was then cut across, exposing the sciatic nerve, as well as the sural, common peroneal and tibial nerves. The common peroneal and tibial nerves were tightly ligated with 5.0 silk and sectioned distal to the ligation, removing $2-4 \mathrm{~mm}$ of the distal nerve stump. Muscle and skin were enclosed in two layers, avoiding any contact with or stretching of the intact sural nerve.

Behavioral assessments were conducted at the following time-points: postoperative week 0 (POW0) (designated as the pretest baseline data), POW1, POW2 and POW3. All rats were sacrificed periodically at POW3. The L4-5 segments of spinal cord were dissected and split into left and right halves from the ventral midline, which were then cut into the dorsal and ventral horn at the level of the central canal. The dorsal horn of rats was used to conduct the following analysis.

Grouping. The rats were randomly divided into 7 groups ( $\mathrm{n}=6$ for each group): sham group, SNI group, saline group (intrathecal injection with $20 \mu 1$ of sterile saline for 3 consecutive days postSNI), PDTC group (intrathecal injection with $2 \mathrm{nmol} / 20 \mu \mathrm{l}$ of PDTC for 3 consecutive days post-SNI), tizanidine group (intrathecal injection with $2.5 \mu \mathrm{g} / 20 \mu \mathrm{l}$ of tizanidine for 3 consecutive days post-SNI), saline + tizanidine group (pretreatment with $20 \mu \mathrm{l}$ of sterile saline at $30 \mathrm{~min}$ before intrathecal injection with $2.5 \mu \mathrm{g} / 20 \mu \mathrm{l}$ of tizanidine for 3 consecutive days post-SNI), and BRL44408 + tizanidine group (pretreatment with $15 \mu \mathrm{g} / 20 \mu \mathrm{l}$ of BRL44408 at $30 \mathrm{~min}$ before intrathecal injection with $2.5 \mu \mathrm{g} / 20 \mu \mathrm{l}$ of tizanidine for 3 consecutive days post-SNI).

Drug administration. Tizanidine $(2.5 \mu \mathrm{g} / 20 \mu \mathrm{l})$, PDTC $(2 \mathrm{nmol} / 20 \mu \mathrm{l})$ and BRL44408 (15 $\mu \mathrm{g} / 20 \mu \mathrm{l}$; Sigma, St. Louis, $\mathrm{MO}$, USA) were diluted in normal saline (NS, $0.9 \% \mathrm{NaCl})$ and loaded into $12.7 \mathrm{~mm} 30$ gauge needle. Under inhalation anaesthesia using isoflurane ( $2 \%$ in oxygen), the rats were injected with drug at the L5-6 interspace.

Mechanical allodynia test. Mechanical allodynia was assessed by measuring the paw withdrawal mechanical threshold (PWMT) in response to a calibrated series of Von Frey hairs (Stoelting, Wood Dale, IL, USA). Each filament was applied five times, and each application lasted for $2 \mathrm{sec}$ with $30 \mathrm{sec}$ interval between trials. A positive response to a filament was indicated by the withdrawal of a hind paw upon application of a particular hair for at least $3 / 5$ consecutive applications. The PWMT was defined as the smallest value of the hair force in grams that elicited positive responses.
Thermal hyperalgesia test. Thermal hyperalgesia was studied by measuring the paw withdrawal thermal latency (PWTL) in response to a radiant heat source. Rats in each group were put on an elevated glass platform, and a radiant heat source (Model 336; IITC Life Science, Woodland Hill, CA, USA) was applied to the plantar surface of the hind paw through the glass plate. We measured the time from onset of radiant heat application to withdrawal of the rat's hind paw, and both hind paws were tested independently with $10 \mathrm{~min}$ interval between trials.

Western blot analysis. The dorsal horns were lysed with icecold lysis buffer. Proteins in the supernatants were quantified by using a BCA kit (Thermo Fisher Scientific, Rockford, IL, USA) and separated with $12 \%$ sodium dodecyl sulfatepolyacrylamide gel electrophoresis (SDS-PAGE). Proteins were transferred onto a polyvinylidene difluoride membrane (Thermo Fisher Scientific), which was then incubated with phosphate-buffered saline (PBS) containing 5\% milk overnight at $4^{\circ} \mathrm{C}$. The membrane was incubated with primary antibodies at room temperature for $3 \mathrm{~h}$, respectively, and then with secondary antibody (both from Abcam, Cambridge, MA, USA) at room temperature for $40 \mathrm{~min}$. Super Signal West Pico Chemiluminescent Substrate kit (Thermo Fisher Scientific) was used to detect signals, according to the manufacturer's instructions. The relative protein expression was analyzed by Image-Pro Plus software 6.0, represented as the density ratio versus glyceraldehyde 3-phosphate dehydrogenase (GAPDH).

Enzyme-linked immunosorbent assay (ELISA). ELISA was conducted to examine the production of inflammatory cytokines in dorsal horns of rats in each group. Rat IL-1 $\beta$ ELISA kit, IL-6 ELISA kit, and TNF- $\alpha$ ELISA kit (all from Biorbyt, Shanghai, China) were used to measure the levels of IL-1 $\beta$, IL- 6 and TNF- $\alpha$, according to the manufacturer's instructions. The optical density at $450 \mathrm{~nm}$ was detected by using Synergy ${ }^{\mathrm{TM}}$ Mx microplate absorbance reader (BioTek, Winooski, VT, USA).

Statistical analysis. Data were expressed as mean \pm standard deviation from three separate experiments. SPSS 19 was used to perform statistical analysis. Student's t-test was conducted when comparing two groups, and one-way ANOVA was conducted when comparing more than two groups. $\mathrm{P}<0.05$ were considered statistically significant.

\section{Results}

SNI induces mechanical and thermal hyperalgesia and inflammatory responses in rats. In the study, we first conducted mechanical allodynia test and thermal hyperalgesia test to determine the PWMT and PWTL in SNI group, and the sham group was used as control. As shown in Fig. 1, PWMT and PWTL showed no difference at different time-points in the sham group. However, the PWMT and PWTL were markedly reduced in SNI group, which lasted for 3 weeks after surgery, indicating that rats in SNI group showed significant mechanical and thermal hyperalgesia.

To further explore the underlying mechanism, we examined the protein expression and secretion levels of proinflammatory cytokines in the dorsal horns using western blot analysis and 
A

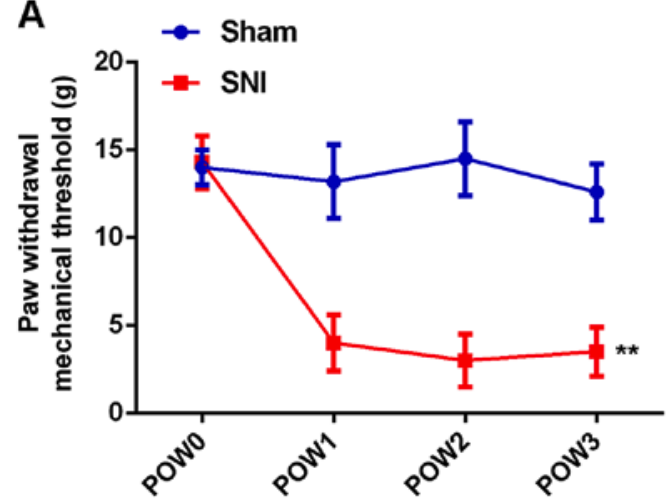

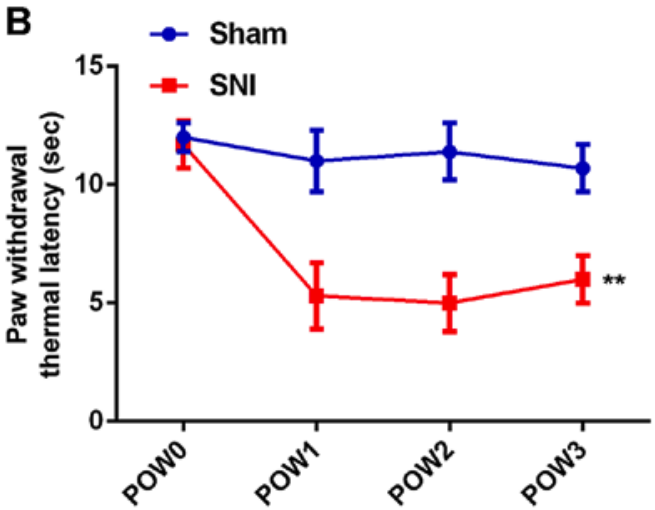

Figure 1. The spared nerve injury (SNI) rat model was constructed, and rats that received sham surgery were used as controls. Behavioral assessments were conducted at the following time-points: postoperative week 0 (POW0), POW1, POW2 and POW3. (A) The paw withdrawal mechanical threshold (PWMT) and (B) paw withdrawal thermal latency (PWTL) were examined. ${ }^{* *} \mathrm{P}<0.01$ vs. sham.

A
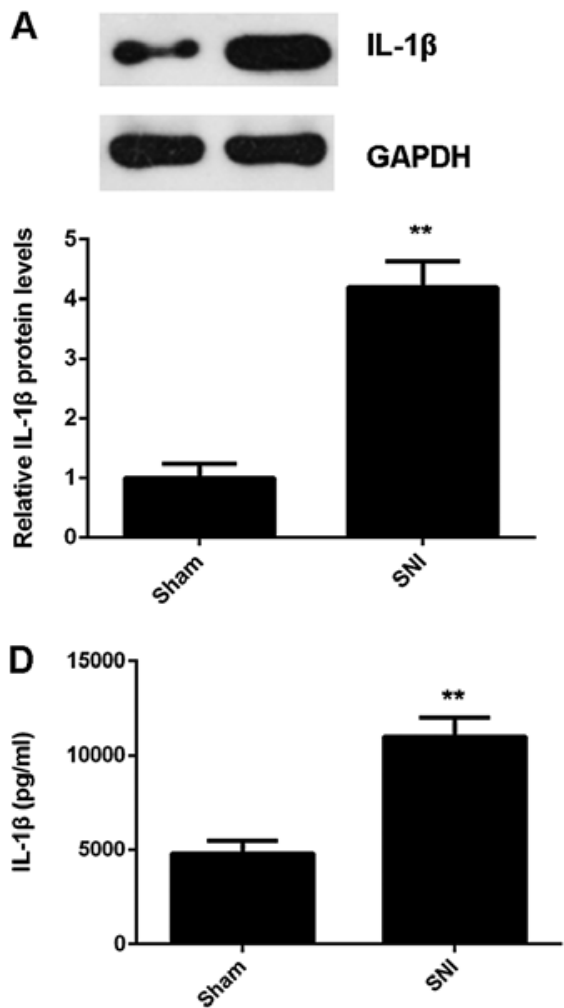

B

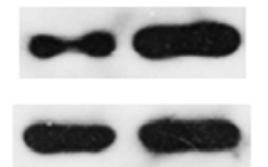

IL-6

GAPDH
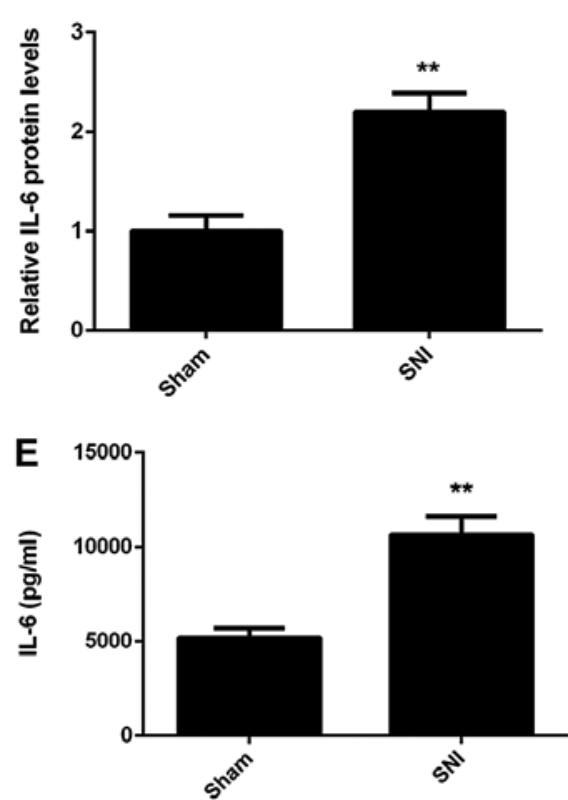

C

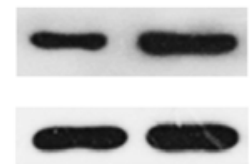

TNF- $\alpha$
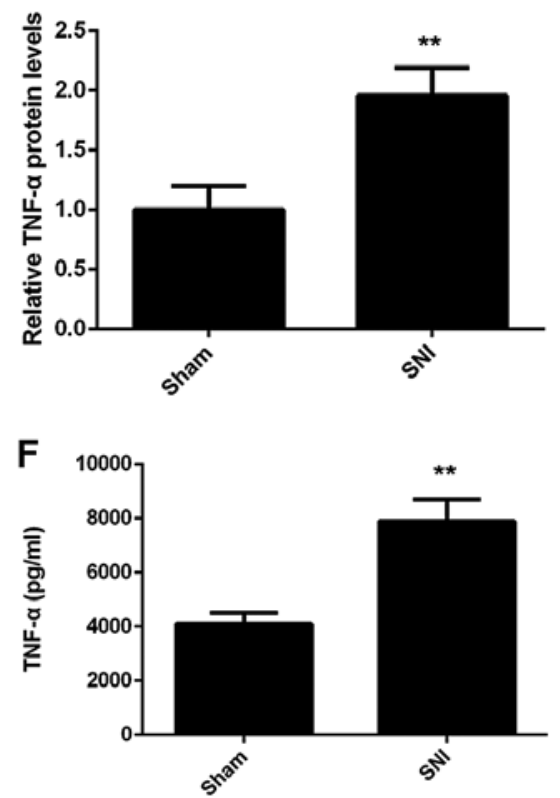

Figure 2. The spared nerve injury (SNI) rat model was constructed, and rats that received sham surgery were used as controls. (A-C) The protein levels of interleukin-1 $\beta$ (IL-1 $\beta$ ), IL-6 and tumor necrosis factor- $\alpha$ (TNF- $\alpha$ ) in the dorsal horns of rats were examined using western blotting. (D-F) The secretion levels of IL-1 $\beta$, IL- 6 and TNF- $\alpha$ in the dorsal horns of rats were examined using enzyme-linked immunosorbent assay (ELISA). ${ }^{* *} \mathrm{P}<0.01$ vs. sham.

ELISA, respectively. As shown in Fig. 2A-C, the protein levels of IL-1 $\beta$, IL-6 and TNF- $\alpha$ were significantly higher in the dorsal horns of rats in the SNI group, when compared with those in the sham group. Consistently, ELISA data showed that the production of these three proinflammatory cytokines were also upregulated in the dorsal horns of rats the SNI group (Fig. 2D-F).

As TLR4/NF- $\kappa \mathrm{B}$ signaling is a key regulator for inflammatory responses (16), we further examined the protein expression of TLR4 as well as the activity of NF- $\mathrm{NB}$. Western blot analysis showed that the protein levels of TLR4 were significantly upregulated in the SNI group compared with the sham group (Fig. 3A). In addition, the phosphorylation levels of $I \kappa B \alpha$ and $\mathrm{NF}-\kappa \mathrm{B}$ p65 proteins were also increased (Fig. 3B and C). These findings indicate that the TLR4/NF- $\kappa \mathrm{B}$ signaling was activated in the SNI group. Accordingly, SNI induces mechanical and thermal hyperalgesia and inflammatory responses in rats, probably through activation of TLR4/NF- $\kappa \mathrm{B}$ signaling.

Inhibition of TLR4/NF- $\kappa B$ signaling attenuates SNI-induced mechanical and thermal hyperalgesia and proinflammatory cytokine production in rats. To further clarify whether TLR4/NF- $\kappa \mathrm{B}$ signaling is essential for the SNI-induced mechanical and thermal hyperalgesia, PDTC, an inhibitor of TLR4/NF- $\mathrm{B}$ signaling, was intrathecally injected into rats for 3 consecutive days after SNI. Intrathecal injection 
A
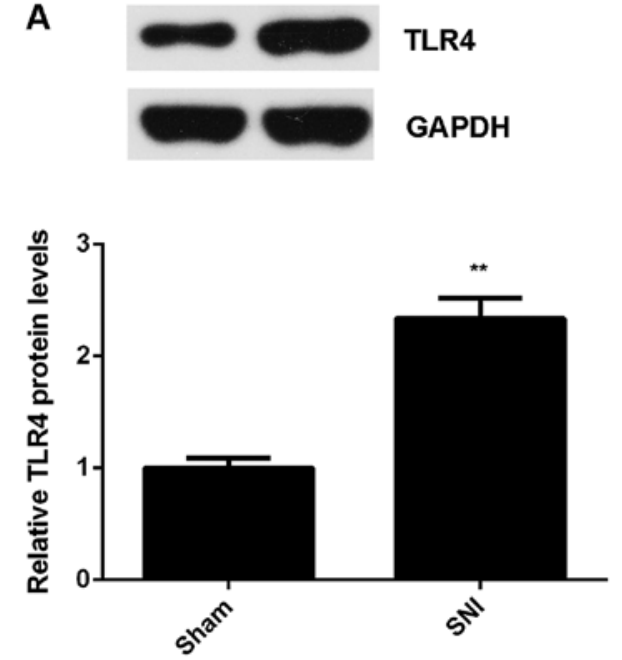

C

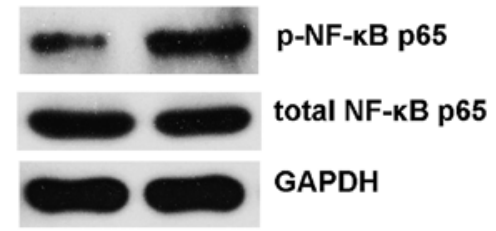

B
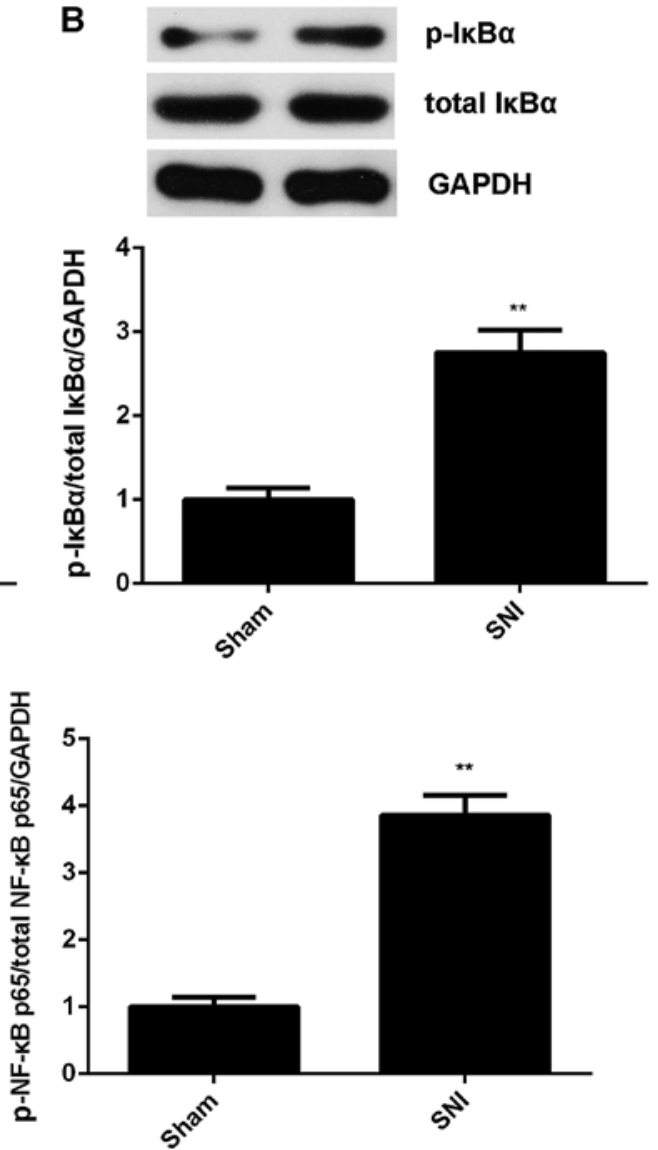

Figure 3. The spared nerve injury (SNI) rat model was constructed, and rats that received sham surgery were used as controls. (A-C) The protein levels of Toll-like receptor 4 (TLR4), phosphorylated I $\kappa \mathrm{B} \alpha$, total $\mathrm{I} \kappa \mathrm{B} \alpha$, phosphorylated nuclear factor- $\kappa \mathrm{B}(\mathrm{NF}-\kappa \mathrm{B}) \mathrm{p} 65$ and total NF- $\kappa \mathrm{B}$ p65 in the dorsal horns of rats were examined using western blotting. ${ }^{* *} \mathrm{P}<0.01$ vs. sham.

with the same amount of sterile saline was used as control. The mechanical allodynia test and thermal hyperalgesia test were performed at different time-points to determine the PWMT and PWTL. As shown in Fig. 4A and B, the PWMT and PWTL were significantly increased in the SNI + PDTC group compared with the SNI + saline group, indicating that treatment with PDTC attenuates SNI-induced mechanical and thermal hyperalgesia.

To further confirm these findings, we examined the activity of TLR4/NF- $\kappa$ B signaling. As shown in Fig. 4C-E, the protein levels of TLR4 as well as the phosphorylation levels of $\mathrm{I} \kappa \mathrm{B} \alpha$ and $\mathrm{NF}-\kappa \mathrm{B}$ p65 proteins were remarkably reduced in the SNI + PDTC group compared with the SNI + saline group, indicating that the treatment with PDTC indeed inhibits the activation of TLR4/NF- $\kappa \mathrm{B}$ signaling in SNI model of rats.

In addition, we found that the protein expression and secretion of IL-1 $\beta$, IL- 6 and TNF- $\alpha$ were also downregulated after PDTC treatment, when compared with the SNI + saline group (Fig. 5). Therefore, our data suggest that the TLR4/NF- $\kappa \mathrm{B}$ signaling is essential for the SNI-induced mechanical and thermal hyperalgesia.

Treatment with tizanidine inhibits SNI-induced mechanical and thermal hyperalgesia in rats. We studied the effects of tizanidine on SNI-induced hyperalgesia in rats. tizanidine was intrathecally injected into rats for 3 consecutive days after SNI. Intrathecal injection with sterile saline was used as control.
Results of mechanical allodynia test and thermal hyperalgesia test showed that the PWMT and PWTL were also increased in the SNI + tizanidine group compared with the SNI + saline group, indicating that tizanidine could attenuate SNI-induced mechanical and thermal hyperalgesia (Fig. 6).

Moreover, western blot analysis and ELISA data indicated that the protein expression and secretion of inflammatory cytokines were also suppressed after tizanidine treatment in SNI rats (Fig. 7). In addition, the activity of TLR4/NF- $\kappa$ B signaling was also downregulated in the tizanidine group compared with the saline group (Fig. 8). Taken together, we demonstrated that treatment with tizanidine inhibits SNI-induced mechanical and thermal hyperalgesia in rats through inhibition of TLR4/NF- $\kappa$ B signaling-mediated inflammatory cytokines production in spinal cord.

Pretreatment with $\alpha 2-A R$ antagonist reverses the inhibitory effects of tizanidine on SNI-induced mechanical and thermal hyperalgesia in rats. As tizanidine is a highly selective $\alpha 2-\mathrm{AR}$ agonist (5), we then performed experiments to clarify whether the inhibitory effect of tizanidine on SNI-induced hyperalgesia was through activation of $\alpha 2-A R$. Pre-intrathecal injection with BRL44408, an $\alpha 2-A R$ antagonist, was performed at $30 \mathrm{~min}$ before injection with tizanidine for 3 consecutive days after SNI. We then examined the PWMT and PWTL at different time-points. As shown in Fig. 9, the PWMT and PWTL were significantly reduced in the BRL44408 + tizanidine group 

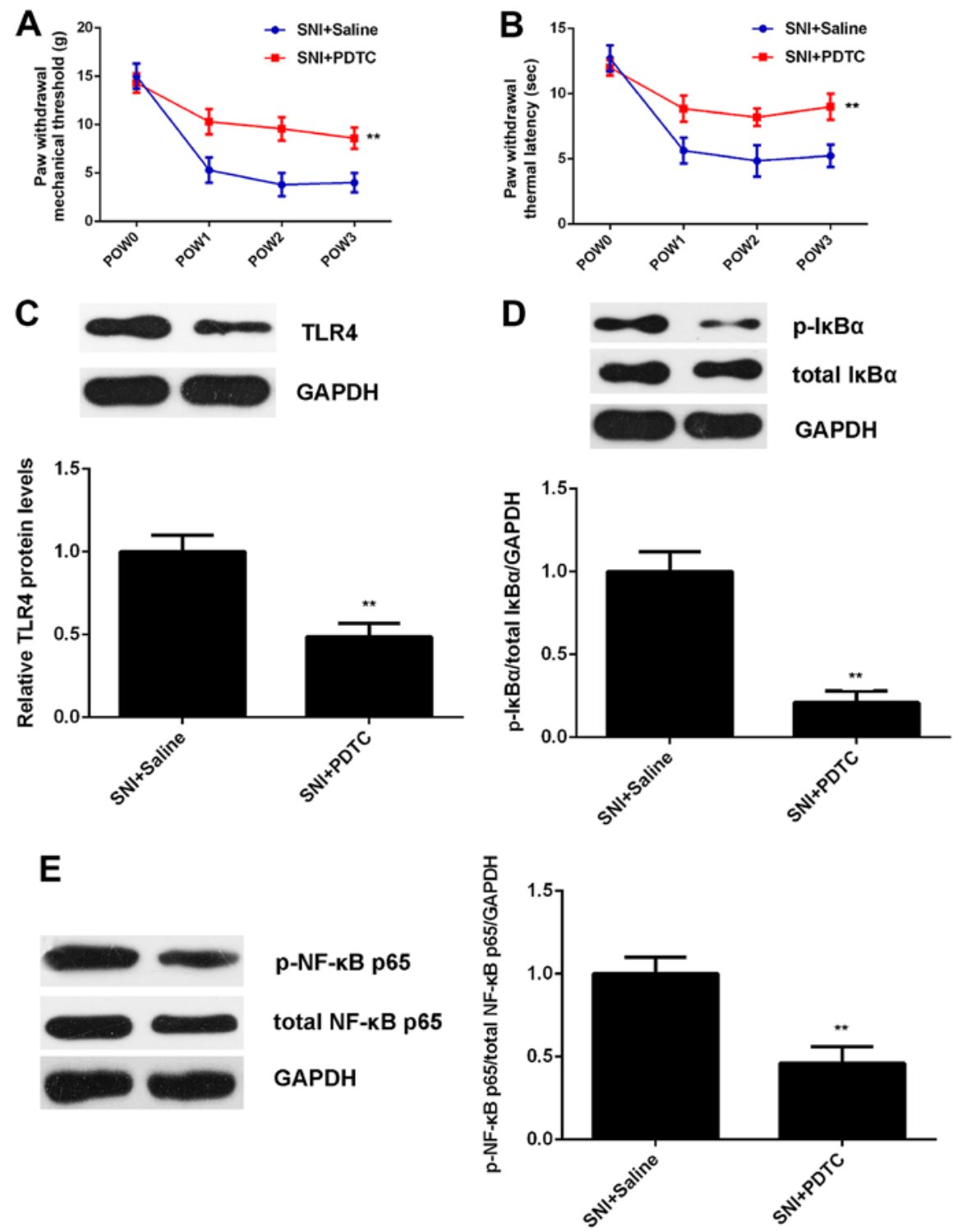

Figure 4. PDTC was intrathecally injected into rats for 3 consecutive days after constructing the spared nerve injury (SNI) rat model. Intrathecal injection with same amount of sterile saline was used as control. Behavioral assessments were conducted at the following time-points: postoperative week 0 (POW0), POW1, POW2, and POW3. (A) The paw withdrawal mechanical threshold (PWMT) and (B) paw withdrawal thermal latency (PWTL) were examined. (C-E) The

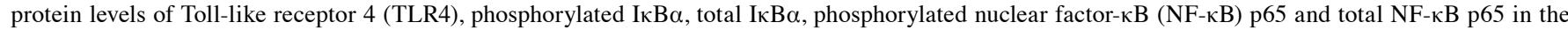
dorsal horns of rats were examined using western blotting. ${ }^{* *} \mathrm{P}<0.01$ vs. SNI + saline.

compared with the saline + tizanidine group, indicating that pretreatment with BRL44408 reverses the inhibitory effects of tizanidine on SNI-induced mechanical and thermal hyperalgesia.

To further confirm these findings, we examined the inflammatory responses in the spinal cord. Our data showed that the protein expression and secretion of IL- $1 \beta$, IL- 6 and TNF- $\alpha$ were increased in pretreatment with BRL44408 (Fig. 10). Moreover, the protein levels of TLR4 and the phosphorylation levels of I $\mathrm{B} \alpha$ and NF- $\kappa \mathrm{B}$ p65 proteins were also upregulated in the BRL44408 + tizanidine group compared with the saline + tizanidine group, indicating that the TLR4/NF- $\mathrm{KB}$ signaling was activated when pretreated with BRL44408 in SNI rats (Fig. 11). According to these above findings, we demonstrated that the inhibitory effect of tizanidine on SNI-induced mechanical and thermal hyperalgesia is directly through activation of $\alpha 2-\mathrm{AR}$ in the spinal cord.

\section{Discussion}

The effect mechanism of tizanidine in neuropathic pain remains largely unknown. Therefore, the present study investigated the effects of tizanidine on neuropathic pain in spared nerve injury (SNI) model of rats, as well as the underlying molecular mechanism. We found that the rats in SNI group showed significant mechanical and thermal hyperalgesia, accompanied by increased production of proinflammatory cytokines in spinal dorsal horn, and the activation of TLR4/NF- $\kappa B$ signaling. The inhibitor of TLR4/NF- $\mathrm{kB}$ signaling PDTC significantly attenuated the SNI-induced mechanical and thermal hyperalgesia and the production of proinflammatory cytokines. Moreover, treatment with tizanidine also attenuated the SNI-induced mechanical and thermal hyperalgesia, suppressed production of the proinflammatory cytokines, and inhibited the activation of TLR4/NF- $\mathrm{B}$ signaling pathway, 
A
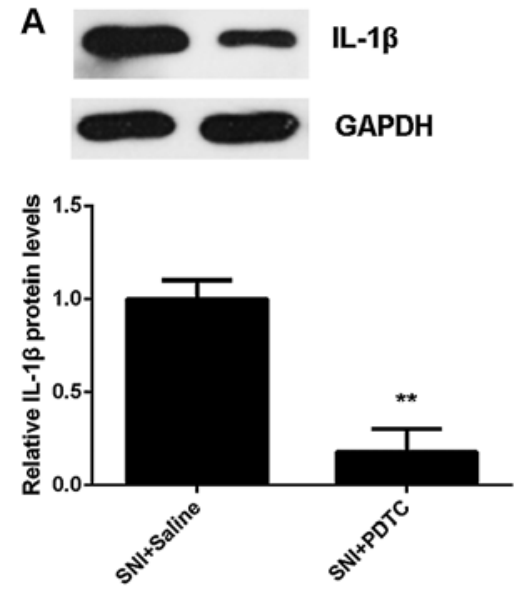

D

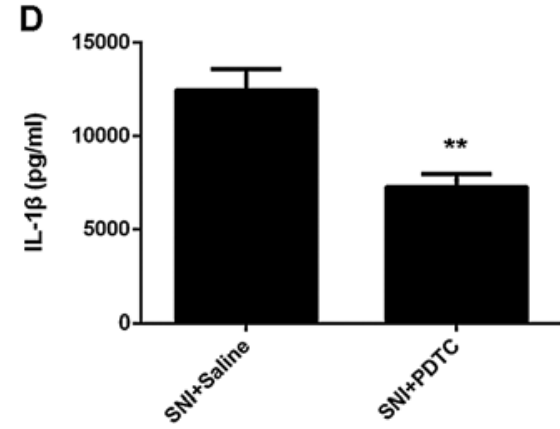

B

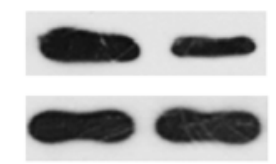

IL-6

GAPDH

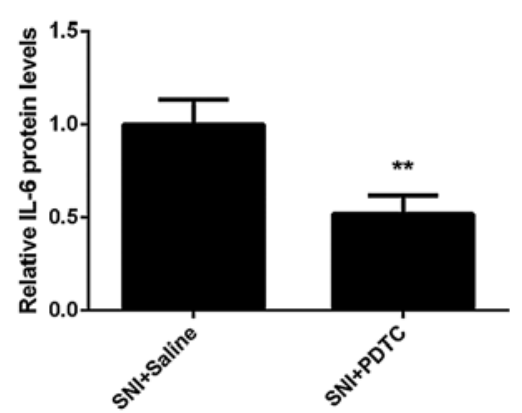

E

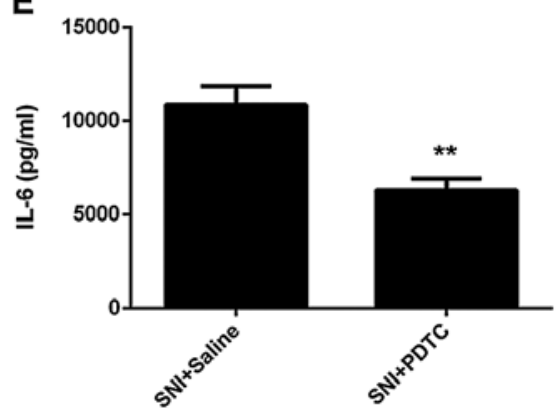

C
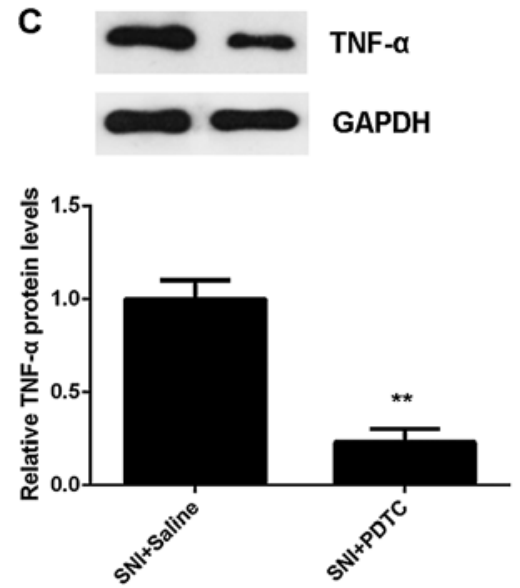

F

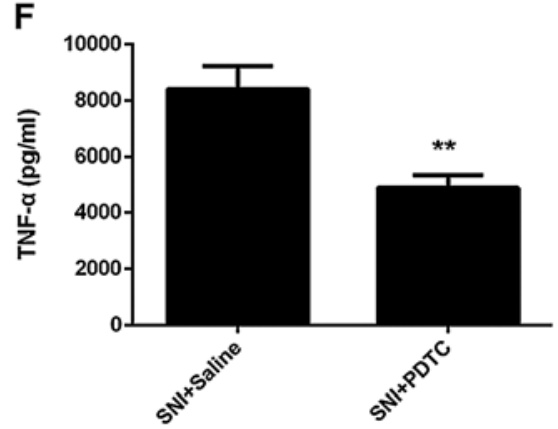

Figure 5. PDTC was intrathecally injected into rats for 3 consecutive days after constructing the spared nerve injury (SNI) rat model. Intrathecal injection with same amount of sterile saline was used as control. (A-C) The protein levels of interleukin-1 $\beta$ (IL-1 $\beta$ ), IL-6 and tumor necrosis factor- $\alpha$ (TNF- $\alpha$ ) in the dorsal horns of rats were examined using western blotting. (D-F) The secretion levels of IL- $1 \beta$, IL- 6 and TNF- $\alpha$ in the dorsal horns of rats were examined using enzyme-linked immunosorbent assay (ELISA). ${ }^{* *} \mathrm{P}<0.01 \mathrm{vs.} \mathrm{SNI}+$ saline.
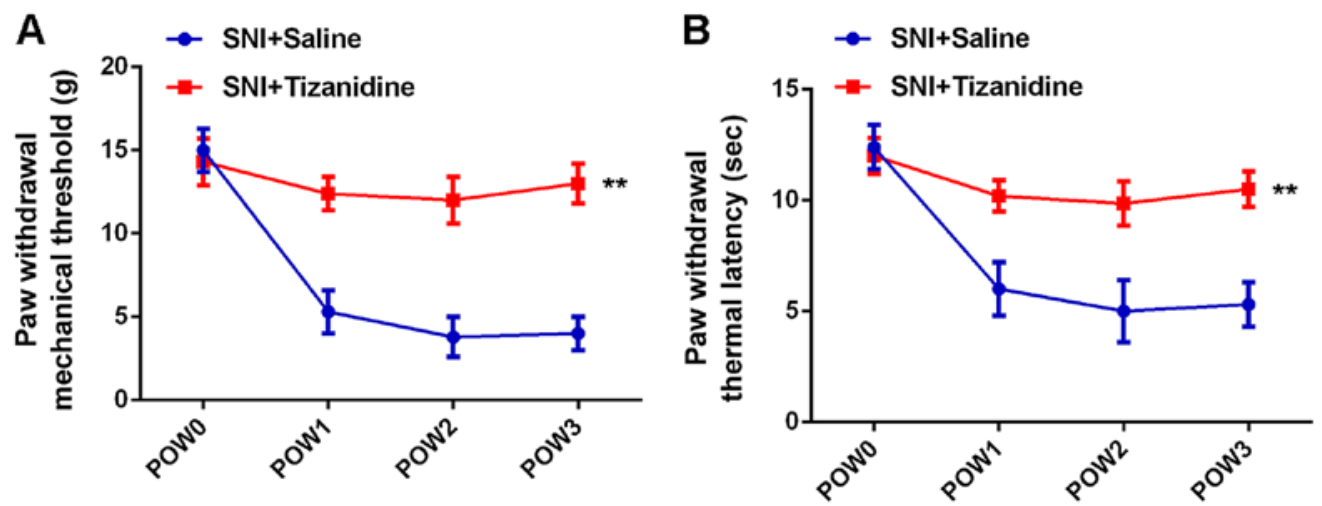

Figure 6. Tizanidine was intrathecally injected into rats for 3 consecutive days after constructing the spared nerve injury (SNI) rat model. Intrathecal injection with same amount of sterile saline was used as control. Behavioral assessments were conducted at the following time-points: postoperative week 0 (POW0), POW1, POW2 and POW3. (A) The paw withdrawal mechanical threshold (PWMT) and (B) paw withdrawal thermal latency (PWTL) were examined. ${ }^{* *} \mathrm{P}<0.01$ vs. SNI + saline.

which could be reversed by pretreatment with BRL44408, a selective $\alpha 2-A R$ antagonist.

Neuropathic pain induces allodynia and hyperalgesia $(20,21)$. SNI is a common model applied for investigating the molecular mechanism underlying peripheral neuropathic pain (22). Previous studies have shown that SNI rats exhibited mechanical and thermal hyperalgesia (23-25), consistent with our findings. Moreover, inflammatory responses have been suggested to participate in the SNI-induced peripheral neuropathic pain (22). Kobiela Ketz et al reported that SNI could cause region-specific activation of macrophages and microglia, and a pro-inflammatory microglial marker was expressed in the spinal cord of SNI rats (22). Recently, Ding et al found that IL-6 was important for the maintenance of SNI-induced neuropathic pain (26). They demonstrated that IL-6 and IL-6R in the red nucleus did not show obvious change at 1 week and 2 weeks after SNI, but was significantly upregulated at 3 weeks after injury (26). Moreover, injection of IL-6 antibody into the red nucleus contralateral to the nerve ligation side at 3 weeks after injury dose-dependently increased the paw withdrawal threshold of rats and alleviated SNI-induced mechanical allodynia (26). Gui et al demonstrated that IL-1 $\beta$ overproduction 
A
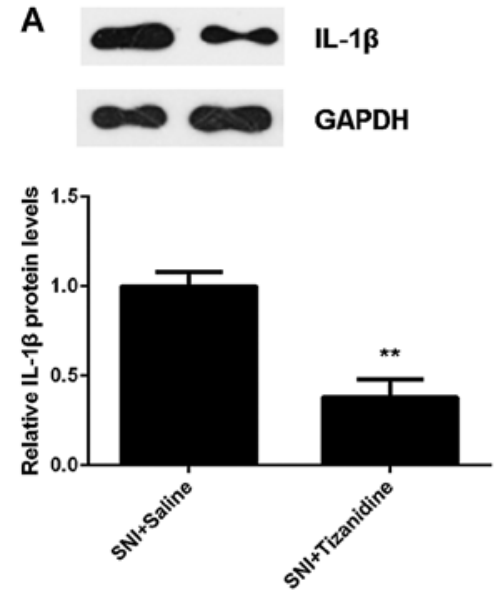

D

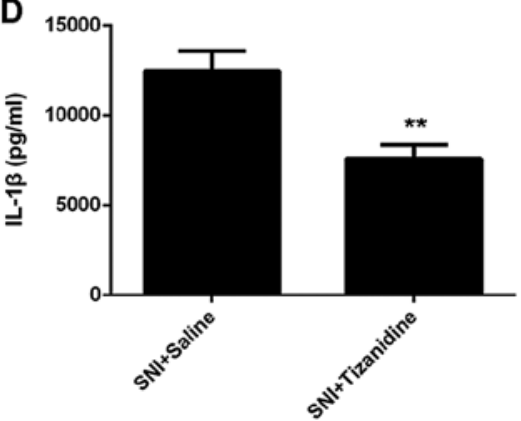

B
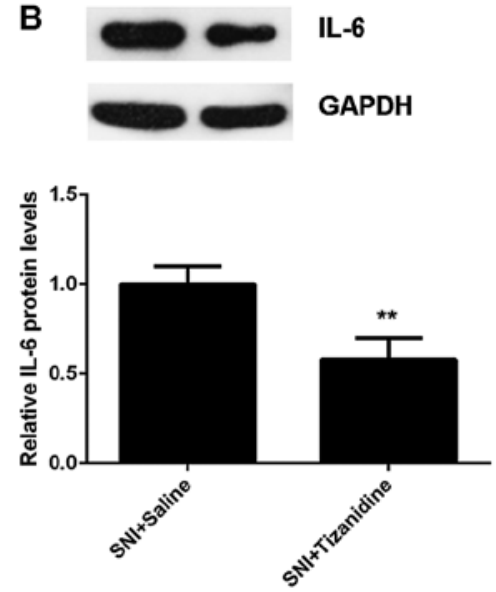

E

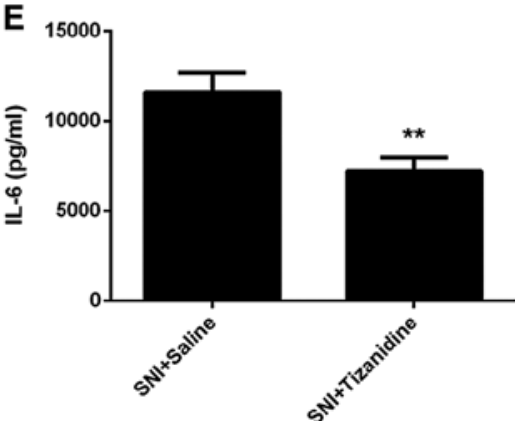

C
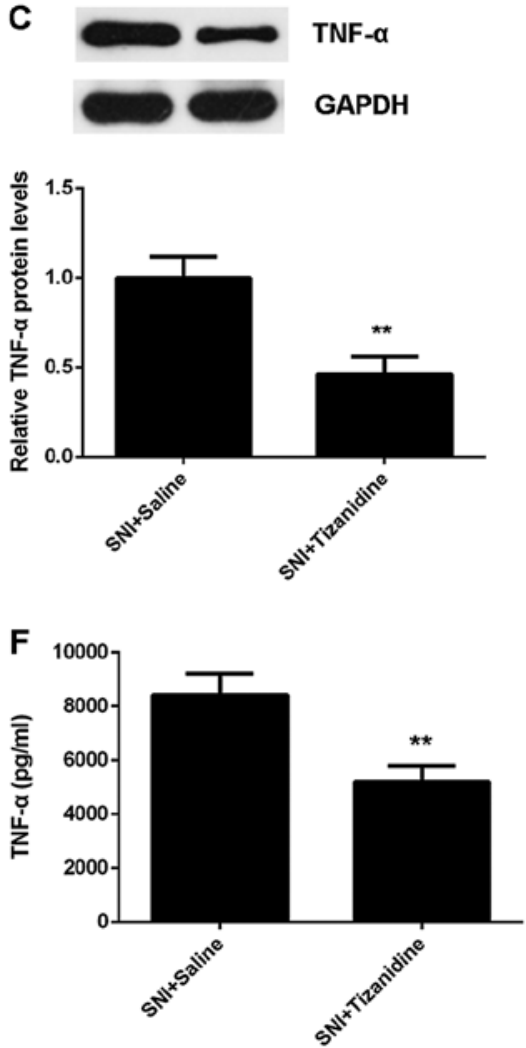

Figure 7. Tizanidine was intrathecally injected into rats for 3 consecutive days after constructing the spared nerve injury (SNI) rat model. Intrathecal injection with same amount of sterile saline was used as control. (A-C) The protein levels of interleukin-1 $\beta$ (IL-1 $\beta$ ), IL-6 and tumor necrosis factor- $\alpha$ (TNF- $\alpha$ ) in the dorsal horns of rats were examined using western blotting. (D-F) The secretion levels of IL-1 $\beta$, IL-6 and TNF- $\alpha$ in the dorsal horns of rats were examined using enzyme-linked immunosorbent assay (ELISA). ${ }^{* *} \mathrm{P}<0.01 \mathrm{vs}$. SNI + saline.
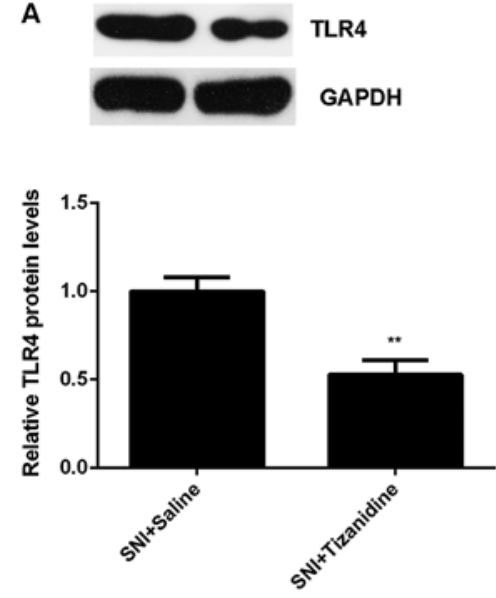

C

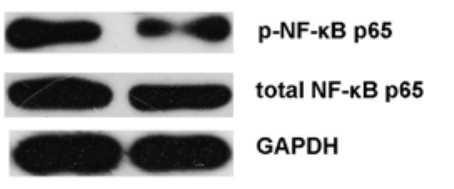

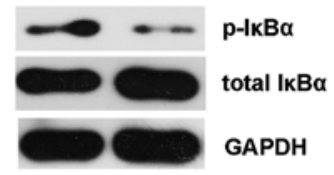
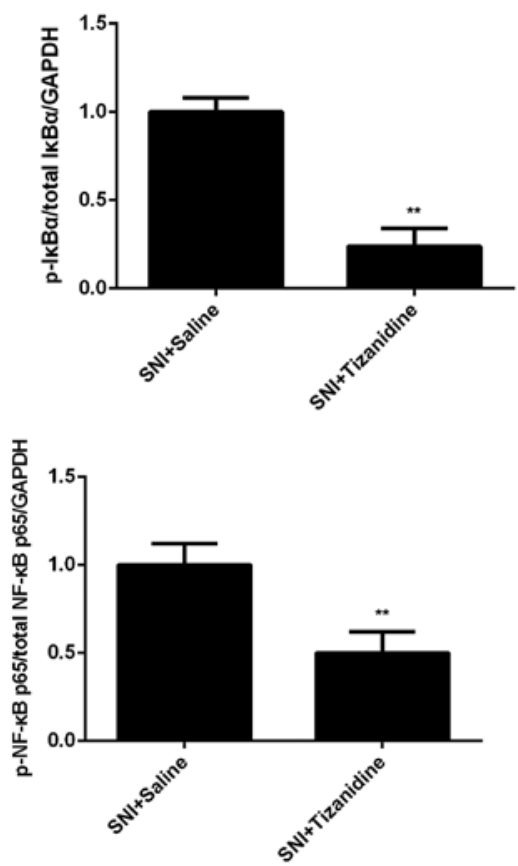

Figure 8. Tizanidine was intrathecally injected into rats for 3 consecutive days after constructing the spared nerve injury (SNI) rat model. Intrathecal injection

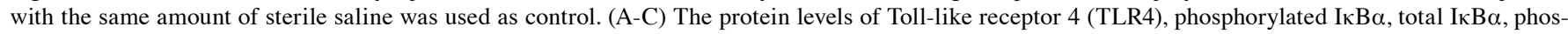

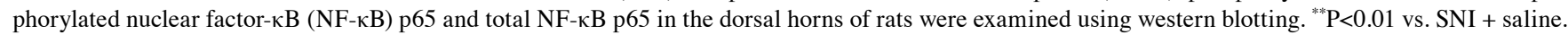



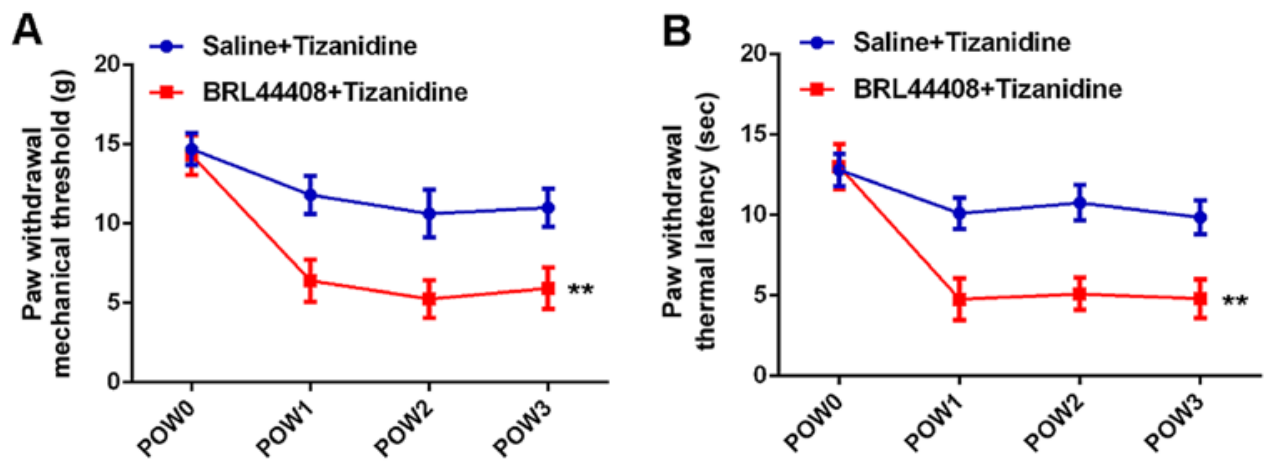

Figure 9. Pre-intrathecal injection with BRL44408 was performed at 30 min before injection with tizanidine for 3 consecutive days after spared nerve injury (SNI). Intrathecal injection with same amount of sterile saline was used as control. Behavioral assessments were conducted at the following timepoints: postoperative week 0 (POW0), POW1, POW2, and POW3. (A) The paw withdrawal mechanical threshold (PWMT) and (B) paw withdrawal thermal latency (PWTL) were examined. ${ }^{* *} \mathrm{P}<0.01$ vs. saline + tizanidine.

A

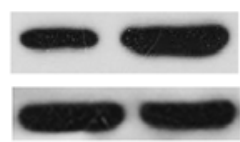

IL-1 $\beta$

GAPDH

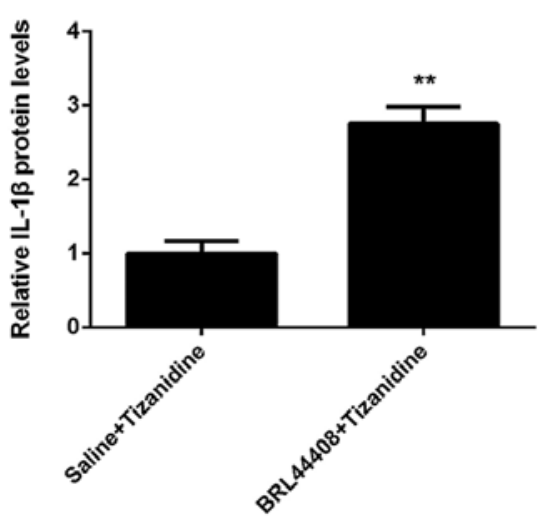

D
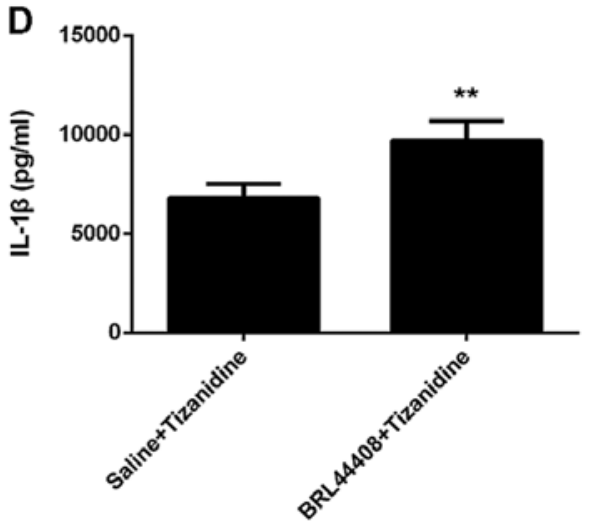

B

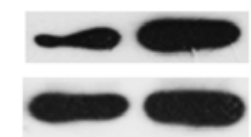

IL-6

GAPDH
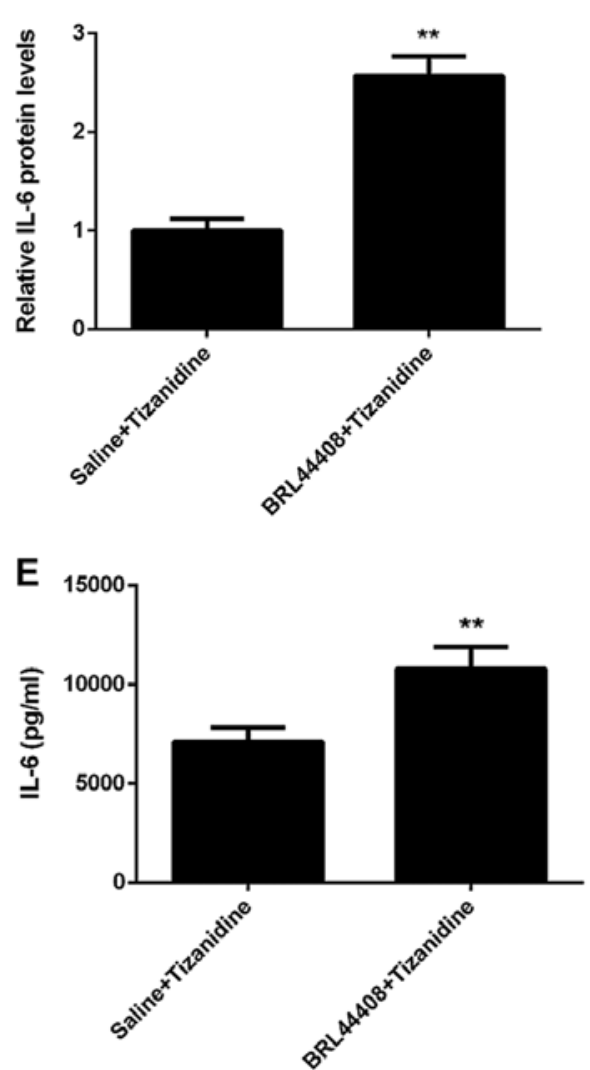

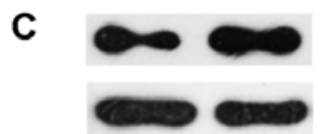

TNF- $\alpha$

GAPDH
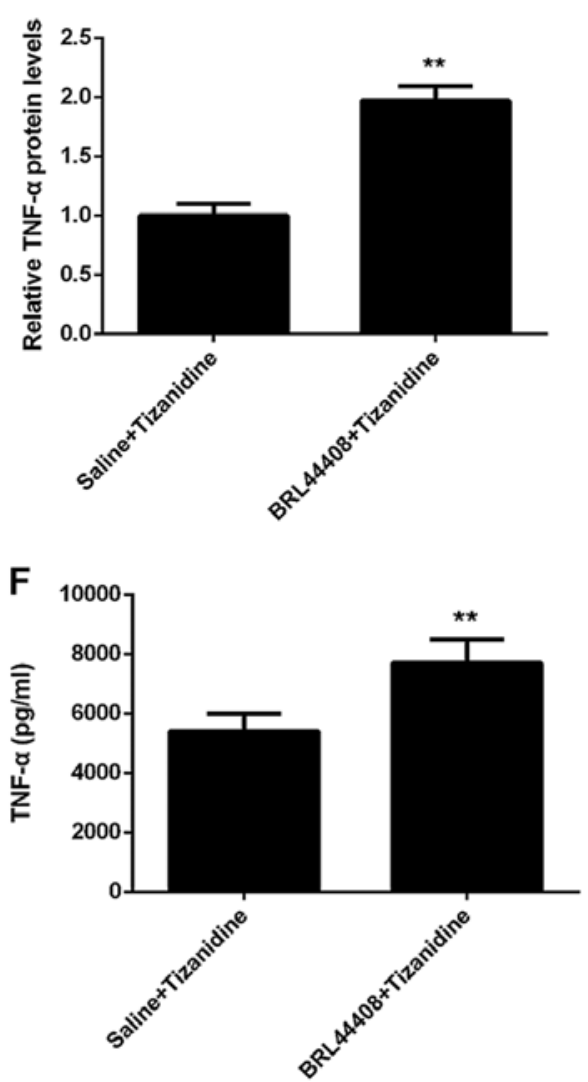

Figure 10. Pre-intrathecal injection with BRL44408 was performed at 30 min before injection with tizanidine for 3 consecutive days after spared nerve injury (SNI). Intrathecal injection with same amount of sterile saline was used as control. (A-C) The protein levels of interleukin-1 $\beta$ (IL-1 $\beta$ ), IL-6 and tumor necrosis factor- $\alpha$ (TNF- $\alpha$ ) in the dorsal horns of rats were examined using western blotting. (D-F) The secretion levels of IL-1 $1 \beta$, IL-6 and TNF- $\alpha$ in the dorsal horns of rats were examined using enzyme-linked immunosorbent assay (ELISA). ${ }^{* *} \mathrm{P}<0.01$ vs. saline + tizanidine.

was a common cause for neuropathic pain following peripheral nerve injury in rodents (27). Besides, TNF- $\alpha$ has been implicated in the development of neuropathic allodynia (28). In this study, we also found that the expression and secretion levels of IL-1 $\beta$, IL- 6 and TNF- $\alpha$ were significantly increased in the spine cord of SNI rats at 3 weeks after injury. As TLR4/NF- $\kappa$ B signaling pathway has been demonstrated to plays a pivotal role in inflammatory responses through the regulation of the expression of proinflammatory cytokines, chemokines and adhesion molecules (14), we further studied its activity. Our data showed that the TLR4/NF- $\kappa \mathrm{B}$ signaling was significantly activated in the spine cord of rats at 3 weeks after SNI. Through using the NF- $\kappa \mathrm{B}$ inhibitor, we further confirmed that the TLR4/NF- $\kappa \mathrm{B}$ signaling is involved in the SNI-induced 
A
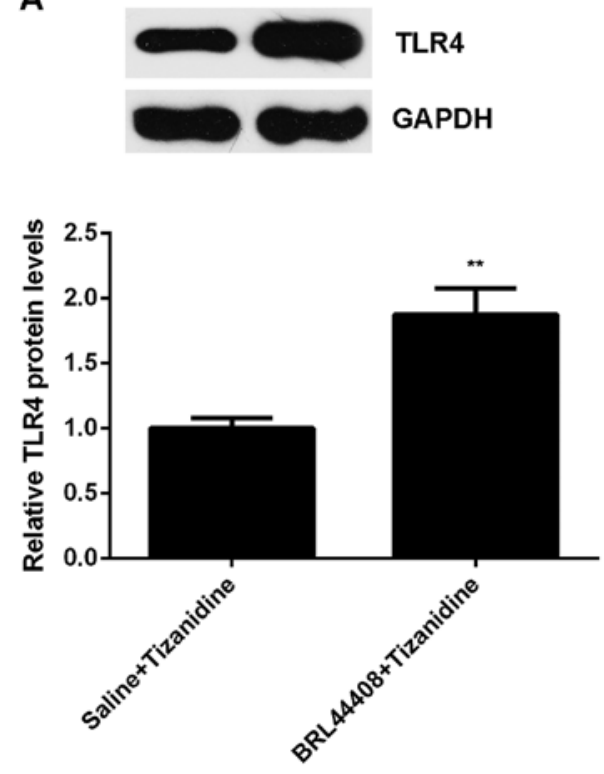

C

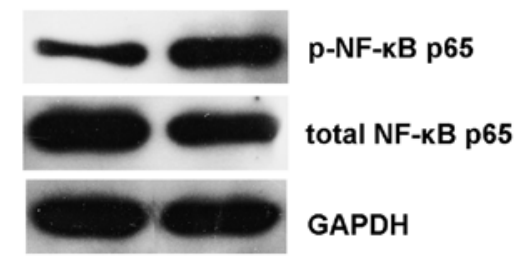

B
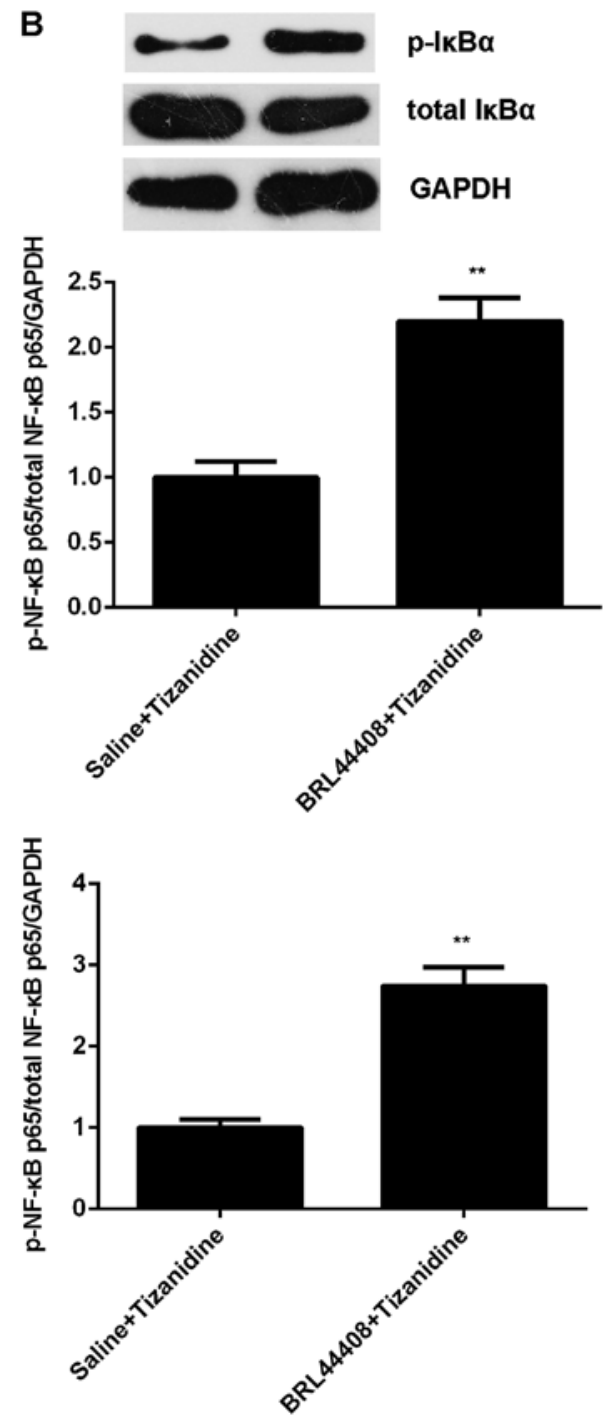

Figure 11. Pre-intrathecal injection with BRL44408 was performed at 30 min before injection with tizanidine for 3 consecutive days after spared nerve injury (SNI). Intrathecal injection with same amount of sterile saline was used as control. (A-C) The protein levels of Toll-like receptor 4 (TLR4), phosphory-

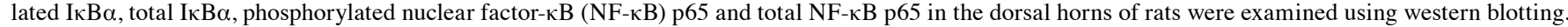
${ }^{* *} \mathrm{P}<0.01$ vs. saline + tizanidine.

mechanical and thermal hyperalgesia and inflammatory responses in spine cord of rats.

It has been reported that the main site where $\alpha 2-A R$ agonists function is the spinal cord $(29,30)$. Tizanidine, a highly selective $\alpha 2-\mathrm{AR}$ agonist, is widely used to treat the spasms, cramping, and tightness of muscles caused by multiple sclerosis, spastic diplegia, back pain, or some other injuries to the spine or the central nervous system $(31,32)$. Previous studies have shown that tizanidine exerts analgesic potential in neuropathic pain $(33,34)$. However, the underlying effect mechanism of tizanidine in neuropathic pain remains largely unknown. In the present study, we for the first time used SNI rat model to investigate the anti-nociceptive effect of tizanidine on SNI-induced neuropathic pain. Our data indicated that intrathecal administration of tizanidine for 3 consecutive days after injury significantly attenuated the SNI-induced mechanical and thermal hyperalgesia. The following mechanism investigation showed that tizanidine could inhibit the SNI-induced production of proinflammatory cytokines as well as the activation of TLR4/NF- $\kappa \mathrm{B}$ signaling pathway in the spine cord. As the TLR4/NF- $\mathrm{B}$ signaling pathway participates in regulating the expression of these proinflammatory cytokines, which are important for the maintenance of SNI-induced neuropathic pain (14,26-28), we suggest that inhibition of TLR4/NF- $\kappa \mathrm{B}$ signaling activation is a potential mechanism through which tizanidine exerts the anti-nociceptive effects in SNI rats. Furthermore, as we found that pretreatment with $\alpha 2-A R$ antagonist significantly reversed the inhibitory effects of tizanidine on the SNI-induced mechanical and thermal hyperalgesia, overproduction of proinflammatory cytokines, and activation of TLR4/NF- $\kappa \mathrm{B}$ signaling, we suggest that the inhibitory effect of tizanidine on SNI-induced neuropathic pain is directly through activation of $\alpha 2-\mathrm{AR}$ in spinal cord.

In conclusion, to our knowledge, this study for the first time demonstrates that tizanidine could attenuate the mechanical and thermal hyperalgesia in SNI model of rats through inhibiting the activation of TLR4/NF- $\kappa$ B p65 pathway, and thus decreasing the production of inflammatory cytokines including TNF- $\alpha$, IL- $1 \beta$ and IL- 6 . These findings highlight the anti-nociceptive effects of tizanidine neuropathic pain. 


\section{Acknowledgements}

Not applicable.

\section{Funding}

No funding was received.

\section{Availability of data and material}

All data generated or analyzed during this study are included in this published article.

\section{Authors' contributions}

WP, YZ, LW and WW performed the experiments and statistical analysis. WP wrote the manuscript. YZ and LL designed the present study and revised the manuscript.

\section{Ethics approval and consent to participate}

This study was approved by Animal Care and Use Committee of People's Hospital of Hunan Province (Changsha, China). Animal experiments were consistent with the National Institutes of Health Guide for the Care and Use of Laboratory Animals.

\section{Patient consent for publication}

Not applicable.

\section{Competing interests}

The authors declare that they have no competing interests.

\section{References}

1. Yang YK, Lu XB, Wang YH, Yang MM and Jiang DM: Identification crucial genes in peripheral neuropathic pain induced by spared nerve injury. Eur Rev Med Pharmacol Sci 18: 2152-2159, 2014.

2. Decosterd I and Woolf CJ: Spared nerve injury: An animal model of persistent peripheral neuropathic pain. Pain 87: 149-158, 2000

3. Yoon SY, Kang SY, Kim HW, Kim HC and Roh DH: Clonidine reduces nociceptive responses in mouse orofacial formalin model: Potentiation by Sigma-1 receptor antagonist BD1047 without impaired motor coordination. Biol Pharm Bull 38 1320-1327, 2015.

4. Li SS, Zhang WS, Ji D, Zhou YL, Li H, Yang JL, Xiong YC, Zhang YQ and $\mathrm{Xu} \mathrm{H}$ : Involvement of spinal microglia and interleukin-18 in the anti-nociceptive effect of dexmedetomidine in rats subjected to CCI. Neurosci Lett 560: 21-25, 2014.

5. Mirbagheri MM, Chen D and Rymer WZ: Quantification of the effects of an alpha-2 adrenergic agonist on reflex properties in spinal cord injury using a system identification technique. J Neuroeng Rehabil 7: 29, 2010.

6. Kawamata T, Omote K, Yamamoto H, Toriyabe M, Wada K and Namiki A: Antihyperalgesic and side effects of intrathecal clonidine and tizanidine in a rat model of neuropathic pain. Anesthesiology 98: 1480-1483, 2003.

7. Davies J and Johnston SE: Selective antinociceptive effects of tizanidine (DS 103-282), a centrally acting muscle relaxant, on dorsal horn neurones in the feline spinal cord. Br J Pharmacol 82: 409-421, 1984

8. Koyuncuŏglu H, Ariciŏglu F, Uresin Y, Dizdar Y and Esin Y: Effects of tizanidine on morphine physical dependence: Attenuation and intensification. Pharmacol Biochem Behav 42: 693-698, 1992
9. Yazicioğlu D, Caparlar C, Akkaya T, Mercan U and Kulaçoğlu H: tizanidine for the management of acute postoperative pain after inguinal hernia repair: A placebo-controlled double-blind trial. Eur J Anaesthesiol 33: 215-222, 2016.

10. Kabayel DD, Ozdemir F, Unlu E, Bilgili N and Murat S: The effects of medical treatment and rehabilitation in a patient with adult tethered cord syndrome in the late postoperative period. Med Sci Monit 13: CS141-CS144, 2007.

11. Roy A, Srivastava M, Saqib U, Liu D, Faisal SM, Sugathan S, Bishnoi $S$ and Baig MS: Potential therapeutic targets for inflammation in toll-like receptor 4 (TLR4)-mediated signaling pathways. Int Immunopharmacol 40: 79-89, 2016.

12. Rocha DM, Caldas AP, Oliveira LL, Bressan J and Hermsdorff HH: Saturated fatty acids trigger TLR4-mediated inflammatory response. Atherosclerosis 244: 211-215, 2016.

13. Zhang J, Xia J, Zhang Y, Xiao F, Wang J, Gao H, Liu Y, Rong S, Yao Y, Xu G, et al: HMGB1-TLR4 signaling participates in renal ischemia reperfusion injury and could be attenuated by dexamethasone-mediated inhibition of the ERK/NF- $\mathrm{BB}$ pathway. Am J Transl Res 8: 4054-4067, 2016.

14. Liu DL, Zhao LX, Zhang S and Du JR: Peroxiredoxin 1-mediated activation of TLR4/NF- $\kappa \mathrm{B}$ pathway contributes to neuroinflammatory injury in intracerebral hemorrhage. Int Immunopharmacol 41: 82-89, 2016.

15. Zhu S, Hu X, Tao Y, Ping Z, Wang L, Shi J, Wu X, Zhang W, Yang H, Nie Z, et al: Strontium inhibits titanium particle-induced osteoclast activation and chronic inflammation via suppression of NF- $\kappa$ B pathway. Sci Rep 6: 36251, 2016.

16. Huang Y, Chen R and Zhou J: E2F1 and NF- $\kappa$ B: Key mediators of inflammation-associated cancers and potential therapeutic targets. Curr Cancer Drug Targets 16: 765-772, 2016.

17. Fuentes E, Rojas A and Palomo I: NF- $\kappa$ B signaling pathway as target for antiplatelet activity. Blood Rev 30: 309-315, 2016.

18. Pujari R, Hunte R, Khan WN and Shembade N: A20-mediated negative regulation of canonical $\mathrm{NF}-\mathrm{\kappa B}$ signaling pathway. Immunol Res 57: 166-171, 2013.

19. Schuster M, Annemann M, Plaza-Sirvent C and Schmitz I: Atypical I $\kappa \mathrm{B}$ proteins - nuclear modulators of NF- $\kappa \mathrm{B}$ signaling. Cell Commun Signal 11: 23, 2013.

20. Dos Reis RC, Kopruszinski CM, Nones CF and Chichorro JG: Nerve growth factor induces facial heat hyperalgesia and plays a role in trigeminal neuropathic pain in rats. Behav Pharmacol 27: 528-535, 2016.

21. Gong SS, Li YX, Zhang MT, Du J, Ma PS, Yao WX, Zhou R, Niu Y, Sun T and Yu JQ: Neuroprotective effect of matrine in mouse model of vincristine-induced neuropathic pain. Neurochem Res 41: 3147-3159, 2016.

22. Kobiela Ketz A, Byrnes KR, Grunberg NE, KasperCE, Osborne L, Pryor B, Tosini NL, Wu X and Anders JJ: Characterization of macrophage/microglial activation and effect of photobiomodulation in the spared nerve injury model of neuropathic pain. Pain Med 18: 932-946, 2017.

23. Ko MH, Yang ML, Youn SC, Lan CT and Tseng TJ: Intact subepidermal nerve fibers mediate mechanical hypersensitivity via the activation of protein kinase $\mathrm{C}$ gamma in spared nerve injury. Mol Pain 12: pii: 1744806916656189, 2016.

24. Tilley DM, Cedeño DL, Kelley CA, Benyamin R and Vallejo R: Spinal cord stimulation modulates gene expression in the spinal cord of an animal model of peripheral nerve injury. Reg Anesth Pain Med 41: 750-756, 2016.

25. Curto-Reyes V, Kirschmann G, Pertin M, Drexler SK, Decosterd I and Suter MR: Neuropathic pain phenotype does not involve the NLRP3 inflammasome and its end product interleukin-1 $\beta$ in the mice spared nerve injury model. PLoS One 10: e0133707, 2015.

26. Ding CP, Xue YS, Yu J, Guo YJ, Zeng XY and Wang JY: The red nucleus interleukin- 6 participates in the maintenance of neuropathic pain induced by spared nerve injury. Neurochem Res 41: 3042-3051, 2016

27. Gui WS, Wei X, Mai CL, Murugan M, Wu LJ, Xin WJ, Zhou LJ and Liu XG: Interleukin-1 $\beta$ overproduction is a common cause for neuropathic pain, memory deficit, and depression following peripheral nerve injury in rodents. Mol Pain 12: pii: 1744806916646784, 2016.

28. Li X, Wang J, Wang Z, Dong C, Dong X, Jing Y, Yuan Y and Fan G: Tumor necrosis factor- $\alpha$ of Red nucleus involved in the development of neuropathic allodynia. Brain Res Bull 77: 233-236, 2008

29. Liu L, Ji F, Liang J, He H, Fu Y and Cao M: Inhibition by dexmedetomidine of the activation of spinal dorsal horn glias and the intracellular ERK signaling pathway induced by nerve injury. Brain Res 1427: 1-9, 2012. 
30. Buerkle $\mathrm{H}$ and Yaksh TL: Pharmacological evidence for different alpha 2-adrenergic receptor sites mediating analgesia and sedation in the rat. Br J Anaesth 81: 208-215, 1998.

31. Malanga G, Reiter RD and Garay E: Update on tizanidine for muscle spasticity and emerging indications. Expert Opin Pharmacother 9: 2209-2215, 2008 .

32. Henney HR III and Chez M: Pediatric safety of tizanidine: Clinical adverse event database and retrospective chart assessment. Paediatr Drugs 11: 397-406, 2009.

33. Ou-Yang HD, Zeng WA, Li Q, He WX, Wang PZ, Lin LL, Zhang ZQ and Liu XG: Effects of intrathecal ouabain and tizanidine injection for treatment of neuropathic pain in rats. Nan Fang Yi Ke Da Xue Xue Bao 28: 1760-1763, 2008 (In Chinese).
34. Leiphart JW, Dills CV and Levy RM: Alpha2-adrenergic receptor subtype specificity of intrathecally administered tizanidine used for analgesia for neuropathic pain. J Neurosurg 101: 641-647, 2004.

(i) $(5)$ This work is licensed under a Creative Commons Attribution-NonCommercial-NoDerivatives 4.0 International (CC BY-NC-ND 4.0) License. 